\title{
Enseigner la rhétorique à l'École de Bruxelles
}

Teaching Rhetoric at "l'École de Bruxelles" [Brussels School]

Emmanuelle Danblon, Lucie Donckier de Donceel, Benoît Sans et Benjamin Sevestre-Giraud

\section{(2) OpenEdition}

Journals

Édition électronique

URL : https://journals.openedition.org/recherchestravaux/4460

DOI : $10.4000 /$ recherchestravaux.4460

ISSN : 1969-6434

Éditeur

UGA Éditions/Université Grenoble Alpes

Édition imprimée

ISBN : 978-2-37747-326-7

ISSN : 0151-1874

Référence électronique

Emmanuelle Danblon, Lucie Donckier de Donceel, Benoît Sans et Benjamin Sevestre-Giraud,

«Enseigner la rhétorique à l'École de Bruxelles », Recherches \& Travaux [En ligne], 99 | 2021, mis en ligne le 08 décembre 2021, consulté le 11 décembre 2021. URL : http://journals.openedition.org/ recherchestravaux/4460; DOI : https://doi.org/10.4000/recherchestravaux.4460

Ce document a été généré automatiquement le 11 décembre 2021.

(c) Recherches \& Travaux 


\title{
Enseigner la rhétorique à l'École de Bruxelles
}

\author{
Teaching Rhetoric at "l'École de Bruxelles" [Brussels School] \\ Emmanuelle Danblon, Lucie Donckier de Donceel, Benoît Sans et Benjamin \\ Sevestre-Giraud
}

1 En 1958, Chaïm Perelman fait paraître son Traité de l'argumentation qu'il signe avec Lucie Olbrechts-Tyteca1. Ils lui donnent le sous-titre de " Nouvelle rhétorique » en hommage explicite à la Rhétorique d'Aristote. Cet événement peut être considéré comme l'acte de naissance de l'approche rhétorique connue aujourd'hui sous l'étiquette d'«École de Bruxelles ». Dans ce traité touffu aux références parfois déroutantes, il faut retenir le cœur du message. L'héritage cartésien qui était celui dans lequel le jeune Perelman, juriste et philosophe, a été formé n'a été d'aucune utilité pour relever le défi intellectuel et politique auquel la société européenne des années 1940 a été confrontée. C'est pourquoi, affirment les auteurs du Traité, il faut retourner à la rhétorique des Anciens, dont Aristote est le premier théoricien, pour récupérer des compétences citoyennes dont la Modernité a cru vouloir débarrasser la société en la rationalisant. Cet enseignement millénaire mis au service de l'éducation avait en effet été évincé au profit de la philosophie et de l'histoire. Il y va donc, aux yeux de Perelman et OlbrechtsTyteca, d'une révolution dans notre conception de la rationalité mise au service d'une éducation à la démocratie et à l'esprit critique. Dans un mouvement qui va à contrecourant de la pensée de l'époque, ce qu'on peut lire entre les lignes, en somme, est l'avertissement suivant : une société démocratique risque de basculer dans la violence et l'obscurantisme, non pas par un excès de rhétorique (lequel excès est incarné par la propagande de masse) mais par une carence en rhétorique.

2 Entrons dans le grain fin des causes présumées de cette carence pour tenter de défendre et d'illustrer une nouvelle fois le besoin de rhétorique, qu'il nous paraît dangereux de réduire à la seule éloquence, nous y reviendrons, tel qu'il se fait à nouveau sentir aujourd'hui, en ce xxI ${ }^{\mathrm{e}}$ siècle déjà largement entamé. Deux notions sont au centre de la pensée de Perelman, qui justifient son ambition de retourner à la rhétorique d'Aristote. La première, éminemment politique, est le statut qu'il faut 
accorder au désaccord. La seconde, liée à la première et, quant à elle, éminemment rhétorique, est le rôle des auditoires.

Commençons par le statut du désaccord. Dans l'approche défendue par Perelman, le désaccord est un fait politique de la démocratie. On pourrait même dire qu'il témoigne de la santé d'une société qui est apte, en toute maturité, à accueillir la diversité des points de vue, tout en se donnant les moyens d'en organiser la circulation dans les débats publics capables de déboucher sur des décisions politiquement utiles et éclairées. Dans cette perspective, le désaccord est un fait de la démocratie qu'il faut pouvoir accepter et organiser. Mais l'héritage positiviste dénoncé par Perelman voit, quant à lui, dans le désaccord le témoin d'incompréhensions réciproques, toujours plus ou moins associées à des erreurs de jugement. Il faut voir là, en filigrane, se dessiner un autre statut du désaccord : celui dont nous avons hérité par la dialectique, ce domaine des raisonnements qui seraient débarrassés du caractère aléatoire, incompétent voire ignorant qui fait le règne de la rhétorique. De fait, dans la vision dialectique du débat, telle qu'on la trouve aujourd'hui représentée dans les courants habermassiens ou celui de la pragma-dialectique, le débat est toujours orienté vers le consensus, par la recherche du meilleur argument, ou par une action collective citoyenne qui chercherait à clarifier les échanges en les débarrassant de tout ce qui pourrait les obscurcir. Dans cette vision, donc, le débat est mis au service de la paix civile, laquelle trouverait son critère dans la résolution du désaccord. Voilà la boucle bouclée : le désaccord, toujours suspect de dégénérer en discorde, serait un mal potentiel à évincer des débats. Mais ce postulat a une conséquence délétère : il érige en idéal de rationalité l'éviction de la diversité des points de vue. Il prétend apprendre au citoyen à communier vers un point de vue unique, garanti par la qualité du raisonnement auquel tout être de raison devrait arriver, s'il est assez éduqué et de bonne foi. On voit alors se dessiner deux conceptions du débat bien éloignées l'une de l'autre. À l'inverse, dans la conception rhétorique du désaccord, celui-ci, assumé comme tel, est mis en forme de telle façon que les différentes perspectives dialoguent entre elles par une structuration et une hiérarchisation des différents points de vue. Ce dialogue ne nous est pas naturel. Il demande un apprentissage technique sur lequel nous reviendrons. Un tel apprentissage, nous le pensons, trouve sa source dans les exercices hérités des Anciens.

Poursuivons par la présence des auditoires. Dans l'idéal de rationalité contenu dans le modèle dialectique, le raisonnement devrait pouvoir se mener en étant au maximum débarrassé de la pression des auditoires: de son caractère forcément multiple, particulier, coloré par des avis personnels, histoires individuelles et communautaires, raisons intimes, etc., toute caractéristique citoyenne qui viendrait mettre en danger une possibilité de vivre ensemble. Conscient de cette situation, le message de Perelman serait le suivant : toute société démocratique est condamnée à subir la persuasion des auditoires particuliers. C'est bien le constat opéré par Aristote qui préconise des stratégies de persuasion adaptées aux situations et aux auditoires. La tension se fait jour rapidement: si les stratégies s'adaptent à chaque nouvelle situation, à chaque auditoire particulier, comment tracer une frontière, un cordon sanitaire en somme, entre persuasion et manipulation? On connaît la réponse de Perelman: il faut concevoir un auditoire universel, principe régulateur qui viendrait subsumer la trop grande pression exercée par les auditoires particuliers, toujours potentiellement porteuse de démagogies. L'auditoire universel se donnerait alors comme le garde-fou de la démocratie pour la protéger contre le basculement vers la dictature, la tyrannie, l'obscurantisme. On le sait pourtant, cette notion a été largement critiquée pour son 
caractère trop abstrait, trop désincarné, trop philosophique, en somme. Il n'est pas le lieu d'entrer dans ce débat. Si l'auditoire universel de Perelman n'est pas une panacée, il contient peut-être en germe une version plus réaliste, plus pratique, plus incarnée, que nous ne désespérons pas de retrouver en initiant les citoyens aux techniques de débat mises au point par les Anciens. C'est du moins le pari que nous faisons au GRAL ${ }^{2}$ depuis quelques années. Retourner à la raison rhétorique théorisée par Aristote demande de mieux comprendre l'intérêt intellectuel et politique des exercices de rhétorique qui permettraient à chacun d'améliorer sa performance à débattre tout en acquérant la capacité à explorer la diversité des opinions, en somme, à apprivoiser le désaccord. C'est ce que nous proposons de développer dans cet article.

\section{Pour une réhabilitation pratique de la rhétorique}

\section{Les techniques d'argumentation mises au point par les Anciens}

Les Anciens avaient imaginé les outils permettant de former à une telle vision de la rhétorique. On sait en effet que dès les débuts de son histoire, dans le cadre de la première sophistique, la rhétorique a fait l'objet d'un enseignement ; celui-ci, dans la mesure où nos sources nous permettent de l'apprécier, s'appuyait sur des exercices : les sophistes avaient vraisemblablement inventé, entre autres, la pratique des dissoi logoi ("discours doubles »), qui consistaient à défendre des points de vue opposés sur une même question et dont nous avons conservé quelques exemples ${ }^{3}$. Sans entrer ici dans les détails d'une longue histoire, quelles que soient les critiques qui ont pu être adressées à la rhétorique, le rôle de certaines personnalités, comme Isocrate ou Aristote, la rareté des sources pour certaines périodes, force est de constater qu'un enseignement a perduré, s'est développé, diversifié et s'est répandu de la période classique au monde grec hellénistique ${ }^{4}$. Il s'est ensuite progressivement installé à Rome, non sans quelques résistances et adaptations, et a continué son expansion sous l'Empire. Et c'est justement à cette période, des premiers siècles de notre ère, que la formation à la rhétorique est relativement bien connue, grâce à diverses sources, qu'il s'agisse de manuels ${ }^{5}$, de recueils de corrigés ${ }^{6}$, d'exemples et d'allusions tirés de la littérature ${ }^{7}$ ou conservés sur papyrus. Auprès d'un rhéteur, les élèves de l'Antiquité étaient d'abord soumis à une série d'exercices de difficulté progressive appelés en grec progymnasmata («exercices préparatoires»): ces exercices allaient d'exercices d'écriture relativement simples (écrire une fable, un récit) à des argumentaires élaborés et souvent appariés (composer un argumentaire pour et/ou contre une proposition de loi fictive), en passant par certains procédés ou types de développement, comme l'éloge, l'ekphrasis (description particulièrement vivante, visant à mettre sous les yeux l'image du sujet représenté) ou encore l'éthopée (imitation dans un discours à la première personne du caractère, ou ethos, d'un personnage dans une situation donnée). Ce parcours impressionne par sa complétude et couvrait l'ensemble des tâches de l'orateur, que ce soit l'inventio (trouver les preuves et les arguments), la dispositio (l'organisation de la matière en différentes parties) ou l'elocutio (style et expression) ; les exercices écrits étaient en outre oralisés, accompagnés de lectures, d'analyses, de reformulations et de réélaborations diverses, permettant d'aborder l'actio (prononciation, gestuelle, performance) et son support, la memoria. En outre, les exercices puisaient abondamment leur matière dans la littérature, la mythologie et l'histoire. Comme leur nom l'indique, les progymnasmata préparaient eux-mêmes à 
l'étape suivante, les déclamations, qui consistaient à produire des discours complets dans des situations censées être les plus proches des causes réelles : la "suasoire" imitait le genre délibératif en conseillant ou en déconseillant une action; la "controverse " consistait quant à elle en une sorte de procès fictif. En somme, ce parcours à la fois rhétorique et littéraire visait à former un citoyen complet, capable d'apprécier, mais aussi d'écrire ou de produire texte ou discours, dans différents genres et n'importe quelle circonstance de la vie publique.

6 Pourquoi le retour de la rhétorique est-il si compliqué ? Malgré les concours d'éloquence, une attention nouvelle portée dans les nouveaux programmes belges et français à l'oral et aux questions d'argumentation dans le discours, l'enseignement de la rhétorique est encore loin d'avoir retrouvé sa pleine légitimité non seulement dans l'enseignement secondaire, mais également à l'université. Nous présenterons ainsi l'enseignement de la rhétorique à l'École de Bruxelles, en nous intéressant d'abord aux critiques que nous avons rencontrées et auxquelles cette discipline technique doit répondre pour exister. D'après notre expérience, ces critiques sont au nombre de trois : l'immoralité, l'inutilité et le danger épistémologique que la rhétorique ferait peser sur la rationalité.

\section{Les critiques adressées au projet de réhabilitation de la rhétorique}

7 Il y aurait d'abord quelque chose de fondamentalement immoral à s'entraîner à argumenter le pour et le contre et, notamment, à faire usage de persuasion, toujours suspecte de manipulation. Sur ce point, la rhétorique est cependant très claire depuis son invention en tant qu'art : son hypothèse de travail, qui fonde sa pédagogie, est de considérer la parole comme une technique, donc comme un outil, dont on peut faire un bon ou un mauvais usage, comme l'illustre le fameux argument du boxeur de Gorgias ${ }^{8}$.

8 L'intérêt de souligner la dimension technique de la rhétorique, dans notre pratique d'enseignement, est de faire le pari que cette compétence persuasive s'enseigne. Tous les étudiants ne deviendront pas des Démosthène ou des Malraux, mais le cours de rhétorique est l'occasion de travailler la compétence oratoire et persuasive en mobilisant les trois sources de l'éloquence théorisée par la tradition rhétorique : les dons naturels (la nature, l'ingenium), les préceptes techniques (la doctrine rhétorique, les praecepta) et l'entraînement pratique (l'exercitatio). N'en déplaise à certaines formations d'art oratoire et d'éloquence, ce n'est pas parce que la rhétorique ne métamorphose pas tout apprenti orateur en showman qu'elle est inutile. Son ambition est en fait bien plus large, puisqu'elle invite plutôt à explorer, par la pratique et à travers des outils techniques, le potentiel persuasif du discours et ses modalités logiques, éthiques et pathétiques.

9 Immorale car techniquement neutre, inutile car modeste, la rhétorique doit également répondre du chef d'accusation de fossoyeuse de la rationalité. Face à l'urgence des fake news, de la " post-vérité ", face à la crise des normes du débat public et médiatique, la rhétorique est le plus souvent présentée comme le poison des sociétés modernes et le symptôme d'un excès de communication, rarement comme une solution. La réponse majoritaire, et sans doute la plus instinctive, à cette crise du langage et de la rationalité semble le plus souvent d'en appeler à plus de normes, à plus de dialectique, de critique et d'analyse. Dans la réflexion pédagogique, on assiste ainsi bien moins à un retour de la rhétorique qu'à celui de la dialectique, son "pendant », dont Aristote avait pourtant 
strictement limité l'usage à la pratique philosophique. Ce renouveau est sensible dans l'essor de la «nouvelle dialectique » et dans l'attrait pour le courant normatif du critical thinking (ou «pensée critique »), qui insiste sur l'urgence d'apprendre à raisonner et à penser en scientifique.

Au GRAL, notre hypothèse de travail est que ce modèle logiciste d'art du discours ne prend en fait pas en compte l'intelligence pratique, le besoin d'entraînement, d'une expérience incarnée, de la production du discours, d'interactions, et de mises en situation pour comprendre et maîtriser le processus de persuasion. Affirmée par le GRAL et récemment par le Manuel de rhétorique de Pierre Chiron, cette proposition voit dans la compétence argumentative et persuasive, acquise par une formation de type rhétorique, simultanément active et réflexive, l'antidote à ce qui peut apparaître comme une perte des repères discursifs et rationnels. Ainsi, c'est parce que l'élève ou l'étudiant saura produire un discours persuasif qu'il sera capable d'en maittriser les effets sur lui-même, dans la position d'un auditeur, en développant un regard proprement rhétorique sur les discours.

Dans ce sens, l'enseignement rhétorique s'appuie sur une conception plus large et plus ouverte de la rationalité - c'est le raisonnable de Chaïm Perelman, opposé au rationnel -, en laquelle réside peut-être tout l'intérêt des Humanités, qui n'ont pas à partager le modèle de rationalité des sciences dites «dures ». La rhétorique est donc capable de prendre en charge cette partie de la formation du citoyen qui, sans jamais abandonner la raison, en explore toutes ses stratifications et s'ouvre notamment à l'intelligence des émotions et de l'intuition.

Ce modèle rhétorique est confronté à une dernière critique, qui ne porte ni sur son épistémologie ni sur ses finalités, mais sur l'opportunité elle-même du retour de la rhétorique: pour des professeurs de français, des enseignants de littérature et d'histoire littéraire, l'idée même d'un retour de la rhétorique peut apparaître saugrenue, puisque la rhétorique serait déjà là, intégrée dans les outils et la méthodologie actuels du littéraire. De ce point de vue, le savoir rhétorique serait encore bien vivant dans l'enseignement littéraire, notamment à travers la stylistique ou l'analyse du discours. Ce serait toutefois réduire drastiquement les ambitions de l'éducation rhétorique à la seule partie de l'élocution (elocutio), qui s'occupe du style, et à une seule activité, le commentaire, d'un corpus de surcroit strictement limité : les textes littéraires. L'ampleur de la tradition et de l'ambition rhétorique nous rappelle en effet la transdisciplinarité essentielle de cet art général du discours persuasif, qui est de toutes les disciplines (cf. Aristote, Rhétorique 1354 a1 ; 1355b 8-9), et l'écart entre une paideia (rhétorique générale), unitaire, et le démembrement actuel de l'ancienne rhétorique en parties restreintes. Réduite à l'analyse du discours ou du texte littéraire, la rhétorique perd l'intérêt de sa démarche active, productive qui vise à faire de l'élève ou de l'étudiant non pas seulement un commentateur, mais un orateur, sujet d'un discours incarné. D'un autre côté, en ne conservant que l'analyse logique, comme c'est le cas dans une approche de pensée critique, ou bien esthétique, comme c'est majoritairement le cas dans l'enseignement littéraire secondaire et universitaire, la tradition rhétorique se replie soit sur son contenu dialectique (l'inuentio, la dispositio) soit sur les figures de style et l'histoire des formes littéraires. Inversement, l'enseignement du discours perdrait également beaucoup à n'être centré que sur l'actio, la performance oratoire et la joute, au service d'une éloquence artificielle ou déconnectée des valeurs et des enjeux démocratiques et humanistes qui le justifient. 


\section{Comment refonder une rhétorique pratique aujourd'hui ?}

\section{Une expérience réalisée dans l'enseignement secondaire}

13 Mais une autre question est de savoir si le parcours imaginé par les Anciens peut encore tenir ses promesses aujourd'hui. C'est ce que nous avons voulu vérifier, de 2013 à 2017, dans le cadre d'un projet scientifique financé par le FRS-FNRS ${ }^{9}$ visant à tester les exercices anciens avec des élèves d'un établissement secondaire situé à Bruxelles. Selon un principe similaire à celui de l'archéologie expérimentale, il s'agissait à la fois de mieux comprendre le fonctionnement des exercices par leur mise en pratique et d'observer leurs effets sur les performances et les capacités des élèves, tout en offrant des savoirs et des savoir-faire réels et utiles. Les résultats de cette expérience, qui ont déjà fait l'objet de diverses publications ${ }^{10}$, ont montré que les exercices anciens permettaient non seulement d'acquérir des compétences techniques, mais également de stimuler le développement de capacités cognitives et relationnelles, telles que l'ouverture d'esprit, l'empathie, l'intelligence émotionnelle ${ }^{11}$ ou la créativité. Les élèves transféraient alors ces nouvelles compétences dans d'autres contextes. L'expérience a donc montré que les exercices anciens pouvaient répondre à des besoins et des publics contemporains, mais également, qu'en respectant les principes qui permettent de fabriquer ces exercices, ceux-ci pouvaient être adaptés à d'autres domaines et problématiques qui dépassent le cadre strictement scolaire.

\section{Un exercice de rhétorique « sur mesure »}

14 Passons à présent à la présentation d'un cas concret. Revenons à l'idée selon laquelle la rhétorique est de toutes les disciplines. Cette idée, nous la reprenons à notre compte et nous tentons de l'incarner, au sein du GRAL, à travers nos chantiers de recherche et les différents exercices pratiques que nous proposons. Dans cette optique, suite aux précédents travaux menés par les membres du GRAL nous avons notamment développé, pour un public constitué d'académiques et d'acteurs du monde social, un exercice autour de la greffe de foie ${ }^{12}$.

Avec cet exercice, nous avions deux ambitions principales : l'une d'ordre politique et l'autre d'ordre théorique. Au niveau politique, nous avons tenté de répondre à la question suivante : comment articuler préoccupations éthiques et médicales dans le cadre d'une prise de décision lorsque les ressources sont limitées ${ }^{13}$ ? Au niveau théorique, il s'agissait pour nous de mettre l'accent sur la tâche d'inuentio et plus globalement, de laisser place à la technique rhétorique pour que celle-ci puisse s'exercer. Ce travail, comme d'autres exercices conçus par l'équipe ${ }^{14}$, s'inscrivait dans une perspective d'adaptation et de réhabilitation des exercices anciens de déclamation. Travailler un type de déclamation (la suasoire) permettait, et permet toujours, à partir de situations prototypiques et fictives, le développement d'une pratique argumentative et rhétorique, en vue du jugement judiciaire ou de la prise de décision politique ${ }^{15}$.

Pour ce faire, nous avons imaginé une situation délibérative fictive au cours de laquelle une décision devait être prise. Telle était la situation inventée: quatre médecins, 
défendant chacun les intérêts de leur patient, doivent se mettre d'accord sur lequel des quatre malades bénéficiera du seul foie disponible ${ }^{16}$ en vue d'une transplantation.

La situation fictive posée, inspirée de la suasoire antique, nous nous sommes attelés à la construction de l'exercice. Or, comme nous l'avons rappelé, si de nombreux exemples d'exercices réalisés par les Anciens nous ont été transmis, nous disposons en revanche de très peu de matériel de référence quant à la construction de ces exercices. La tâche de créer un exercice rhétorique doit donc se faire à rebours, en partant des résultats et des objectifs espérés, pour aboutir à un cadre spécifique qui permette aux participants d'atteindre les buts donnés ${ }^{17}$.

Dans le cas de l'exercice de la greffe de foie, nous le disions, il s'agissait pour nous de revaloriser le recours à la rhétorique pour la prise de décision politique. Mais il s'agissait aussi d'une opportunité pour développer la capacité de chacun à chercher des arguments (inuentio). Concrètement, cela implique qu'une attention particulière ait été accordée à la notion de lieux ${ }^{18}$ (domaines de la réalité à exploiter pour traiter un sujet donné, ensemble de catégories disponibles pour l'argumentation) : à partir des lieux du délibératif, nous avons imaginé des petits récits de vie permettant aux participants - incarnant les médecins - d'ancrer et d'argumenter leur position. Ces brefs récits de vie ont été construits selon un principe de compensation, laissant expressément place à l'ambiguïté sur certains points et portant une attention particulière à d'autres détails. De la sorte, les lieux du délibératif, inspirés de la théorie de Quintilien ${ }^{19}$, étaient incarnés par les différents patients mis en jeu. Notons également que, dans une même optique, certains éléments ont été neutralisés pour les bienfaits de l'exercice, nous pensons par exemple à la compatibilité et la disponibilité de l'organe, mais également au critère de la temporalité (la même urgence était requise pour tous les patients). De fait, la majeure difficulté dans la construction de l'exercice résidait dans la tâche de rédaction des profils des différents patients. En effet, nous devions nous assurer, comme le laissent penser certains manuels des Anciens, que l'on propose aux participants des situations argumentatives équitables et équilibrées ${ }^{20}$.

19 Au-delà de la tâche spécifique d'inuentio que nous visions, pour la construction de ces quatre brefs récits de vie, et dans le cadre général de l'exercice, nous avons également cherché à attirer l'attention des participants sur les trois preuves techniques de la persuasion (ethos, pathos et logos). Pour ce faire, nous avons distillé au sein de l'exercice, parmi les différentes présentations des patients, des éléments pouvant servir de pistes pour la construction de ces trois preuves au sein des discours. À titre d'exemple, pour ce qui est de l'ethos, nous avons insisté sur le fait que les participants incarnaient des médecins, qu'ils devaient donc prononcer le discours en faisant comme s'ils étaient des professionnels de la santé (on s'inspire ici de l'exercice antique de la prosopopée qui consiste en l'imitation d'une personne/d'un personnage donné, à travers un discours à la première personne); pour le pathos, nous avons veillé à ce que les participants puissent s'appuyer sur certains détails (et parfois préjugés) de la vie des patients pour pouvoir susciter différentes émotions à l'égard de «leur " patient; enfin, pour le logos, nous avons proposé aux participants, nous l'expliquions ci-dessus, de mobiliser les lieux du délibératif dans la construction de leur argumentaire. De fait, les catégories argumentatives que sont les lieux ont été mobilisées à deux niveaux dans le cadre de cet exercice : elles ont été pensées comme outil heuristique pour la construction des profils des patients, mais elles ont aussi été présentées aux participants comme outils heuristiques pour la recherche d'arguments, tout en sachant bien qu'a priori, des 
éléments ayant trait à chaque lieu étaient disponibles parmi les informations dont les participants disposaient pour construire leur argumentation. La mobilisation des lieux sur ces deux plans nous a donc permis de mettre les participants dans une posture active double : une posture de production (dans la construction de leur argumentation) et une posture d'analyse (dans la lecture des différents brefs récits de vie des patients avec la grille des lieux à leur disposition). Cette double posture participe du développement d'un regard réflexif sur la pratique argumentative de chacun ${ }^{21}$.

Nous le disions, ce type d'exercice n'est pas neuf, les exercices de déclamation étaient déjà pratiqués par les Anciens et les catégories des lieux y étaient déjà mobilisées. Nous ne prétendons donc pas les réinventer. Cependant, à travers ces pratiques pédagogiques nous soutenons que la rhétorique antique est adaptable à un cadre de vie actuel et surtout, qu'elle est encore pertinente pour appréhender et aborder les enjeux de nos sociétés contemporaines; en d'autres mots, la rhétorique peut encore répondre à certains de nos besoins actuels. Cela rejoint notre postulat de départ : cet exercice a été pensé pour répondre à un objectif politique et à un objectif théorique, ces deux éléments se rencontrant bien évidemment.

21 En termes politiques, ce type d'exercice souligne l'importance et la complexité du processus décisionnel en démocratie: la décision politique ne se limite pas à des raisonnements logiques ${ }^{22}$. Cela sous-entend une conception de la rationalité plus souple et flexible, incluant les trois preuves rhétoriques : le logos bien évidemment mais aussi le pathos et l'ethos ${ }^{23}$. Ces considérations politiques rejoignent les objectifs théoriques exposés précédemment : permettre l'exercice de la technique et, à travers celle-ci, le développement d'un regard réflexif sur nos différentes pratiques argumentatives. Si l'on prend un peu de hauteur, exercer la technique rhétorique contribuerait au développement de l'esprit critique. Pratiquer la critique - définie comme la capacité de l'esprit à juger un être, une chose à sa juste valeur après en avoir discerné les mérites et les défauts - nécessite la suspension temporaire du jugement, et ce, afin d'explorer ce qu'il y a de raisonnable dans une position opposée à la nôtre ${ }^{24}$. C'est précisément en ce sens qu'un exercice de rhétorique tel que nous le pratiquons permet d'allier intérêts politiques et théoriques. La suspension temporaire du jugement et l'exploration des différents points de vue disponibles, outre le fait de participer à la lutte contre un certain dogmatisme spontané, soutiennent l'idée qu'en démocratie, il existe toujours au minimum trois points de vue raisonnables sur une question donnée et que c'est pour cela, justement, qu'il est nécessaire de délibérer.

Promouvoir le modèle de l'enseignement rhétorique, c'est ainsi non seulement s'inscrire dans la riche tradition humaniste de formation au discours, mais aussi refuser l'évolution antirhétorique des Humanités elles-mêmes. Un enseignement renouvelé de la rhétorique, comme nous le proposons, invite en effet à considérer la fragmentation progressive de disciplines littéraires comme l'histoire, la littérature et la philosophie, chacune régnant sur un domaine respectif. Au terme de cette présentation de la rhétorique dans la tradition pratique et théorique de l'École de Bruxelles, il est en effet étonnant de constater que l'enseignement linguistique et littéraire a suivi l'évolution continue des sciences modernes vers une spécialisation croissante, au point de perdre le caractère complet et unitaire de la tradition d'enseignement rhétorique qui fondait, 
précisément, le modèle antique d'une éducation générale par l'activité littéraire, la paideia.

Comme l'a pourtant rappelé Chaïm Perelman, « celui qui argumente ne s'adresse pas à ce que l'on considère comme des facultés, telle la raison, les émotions, la volonté. L'orateur s'adresse à l'homme complet ${ }^{25}$ " et c'est bien cet homme complet que la tradition humaniste d'enseignement a souhaité former par la pratique rhétorique. En ce sens, l'héritage antique de la rhétorique, unissant les savoirs et les disciplines particulières par la maîtrise et la pratique du discours à finalité politique, mérite en effet d'être rappelé et confronté au morcellement actuel des anciennes Humanités. Renouer avec la pratique d'un enseignement rhétorique, comme nous le proposons, est ainsi une invitation à retrouver la profonde unité des arts du discours et les finalités politiques de la paideia rhétorique antique, aussi diverses que furent ses formes et ses réalisations tout au long de cette longue tradition.

\section{ANNEXES}

\section{Exercice de la greffe de foie}

Atelier de rhétorique

Mardi 4 septembre 2018

GRAL

Groupe de recherche en Rhétorique et Argumentation Linguistique

Université Libre de Bruxelles

Exercice délibératif : "La greffe de foie »

\section{Consignes}

- Les participants sont organisés en quatre groupes équitables, chaque groupe désigne un porte-parole.

- Chaque groupe incarne un médecin devant défendre, devant un comité décisionnel, un patient en attente pour une greffe de foie.

- Le temps de préparation de l'exercice est de 40 minutes; chaque discours doit comporter un exorde, une narration et une péroraison. De plus, chaque discours doit contenir au minimum deux arguments, relatifs à deux lieux différents.

- Le temps de parole accordé à chaque médecin est de 3 minutes.

- Après défense des cas par les médecins, le jury doit accorder sa préférence.

- Un temps est réservé pour la discussion avec les participants, ce temps est dédié à un retour technique sur l'exercice réalisé.

\section{Présentation du profil des patients}

Quatre patients présentent une insuffisance hépatique terminale, une affection qui est irréversible et mortelle. Le seul traitement susceptible de les sauver est la greffe de 
foie. Mais seul un foie compatible avec chacun d'eux est disponible. Le profil des patients ainsi que leur espérance de vie objective sans greffe ont été établis :

- Isabelle, fonctionnaire de 38 ans est mère de deux enfants de 7 et 10 ans, elle souffre d'une maladie auto-immune. Le risque que cette maladie récidive après la greffe est difficile à apprécier. Suite au départ inattendu de son mari avec une autre femme, elle a arrêté de prendre ses médicaments et la fonction de son foie s'est rapidement dégradée. Sans greffe de foie, son espérance de vie est estimée à un mois.

- Pierre, 67 ans, retraité, vit actuellement dans une habitation supervisée par l'hôpital où il a demandé à être hospitalisé il y a deux ans pour se défaire de quinze ans d'alcoolisme. Il n'a plus consommé une goutte d'alcool depuis vingtcinqmois et s'investit en tant que bénévole depuis plusieurs mois. Malheureusement, les lésions sur son foie étaient déjà trop avancées. Il espère reprendre prochainement contact avec sa fille de 35 ans, qu'il n'a plus revue depuis plusieurs années, et est très apprécié des personnes avec qui il travaille. Sans greffe de foie, son espérance de vie est estimée à six mois.

- Françoise, 53 ans, célibataire et sans enfant, est une chercheuse de réputation internationale qui dirige une équipe de recherche sur l'optimisation d'un nouveau traitement permettant de lutter contre la leucémie. Les résultats des premiers tests sont encourageants, et le traitement va bientôt pouvoir être testé sur de vrais patients. Elle souffre aujourd'hui d'un cancer du foie et une transplantation constitue son seul espoir de survie. Françoise souffre par ailleurs d'une malformation cardiaque qui augmente significativement le risque de l'anesthésie. Sans greffe de foie, son espérance de vie est estimée à trois mois.

- Hugo, 17 ans, élève en $6^{\mathrm{e}}$ année de l'enseignement secondaire, est l'aîné d'une fratrie de quatre enfants. Il prépare son examen d'entrée pour l'École polytechnique. Ses parents sont divorcés depuis peu et il vit actuellement chez sa mère, souvent en déplacement. Depuis deux ans, il a l'habitude d'organiser des fêtes chez lui, avec des amis lors de ses absences. Lors de ces soirées, il consomme beaucoup d'alcool. Ce problème de consommation importante et répétée d'alcool n'est perçu ni par Hugo ni par son entourage, il n'a donc jamais été pris en charge. $\mathrm{Au}$ cours de l'une de ces soirées, il a été emmené d'urgence à l'hôpital après avoir bu une quantité importante d'alcool en très peu de temps. Il souffre d'une insuffisance hépatique hyper aiguë et a besoin d'une greffe de foie. Sans greffe de foie, son espérance de vie est estimée à six semaines.

Les médecins délibèrent pour savoir qui aura la priorité pour la greffe. Chaque médecin défend son patient du mieux qu'il peut.

\section{Présentation des lieux à disposition des participants}

Selon Quintilien (III, 8), les lieux du délibératif sont les suivants :

Lieux (lat. loci) 


\begin{tabular}{|l|l|}
\hline Utile (registre pragmatique) & \\
Utile (utile, bénéfique) & \\
Facile (aisé à réaliser, faisable) & \\
Tutum (sûr, sans risque) & Honestum (registre éthique et moral) \\
Opportunum (opportun) & Justice, équité, mérite, clémence, gloire, etc. \\
Necessarium (nécessaire) & (valeurs) \\
$\begin{array}{l}\text { Possibile (possible) } \\
\text { (La règle) }\end{array}$ & \\
\hline
\end{tabular}

\section{NOTES}

1. C. Perelman \& L. Olbrechts-Tyteca, Le Traité de l'argumentation. La nouvelle rhétorique, Paris, Presses Universitaires de France, 1958.

2. Groupe de recherche en Rhétorique et Argumentation Linguistique de l'Université libre de Bruxelles, sous la direction scientifique de la professeure Emmanuelle Danblon.

3. A. Laks \& G. Most, Les débuts de la philosophie. Des premiers penseurs grecs à Socrate. Textes réunis et traduits par A. Laks et G. Most, Paris, Fayard, 2016, p. 1519-1534.

4. L. Pernot, La rhétorique dans l'Antiquité, Paris, Librairie Générale Française, 2000, p. 82-114.

5. Ceux d'Ælius Théon (fin du $\mathrm{I}^{\mathrm{er}}$ siècle ou $\mathrm{II}^{\mathrm{e}}$ siècle apr. J.-C.), Pseudo-Hermogène (III ${ }^{\mathrm{e}}$ siècle apr. J.C.), Aphthonios (IV siècle apr. J.-C.) et Nicolaos ( $\mathrm{V}^{\mathrm{e}}$ siècle apr. J.-C.). Voir G. Kennedy, Progymnasmata. Greek Textbooks of Prose Composition and Rhetoric. Translated with Introduction and Notes, Atlanta, Society of Biblical Literature, 2003 ; M. Patillon, Ælius Théon. Progymnasmata. Texte établi et traduit par M. Patillon, Paris, CUF, 2002 ; M. Patillon, Corpus Rhetoricum, t. 1, Préambule à la rhétorique. Aphthonios, Progymnasmata. Pseudo-Hermogène, Progymnasmata. Texte établi et traduit par M. Patillon, Paris, CUF, 2008.

6. Comme les modèles attribués au rhéteur Libanios, ou ceux de Sévère d'Alexandrie et Choricios de Gaza.

7. On citera notamment les biographies de rhéteurs de Suétone ou Philostrate, la correspondance de Libanios et de Fronton. Les romans grecs et latins semblent également très influencés par la pratique et la formation rhétoriques.

8. Platon, Gorgias $465 \mathrm{~b}$.

9. Fonds de la Recherche Scientifique, anciennement Fonds National de la Recherche Scientifique.

10. J. Dainville \& B. Sans, "Teaching Rhetoric Today: Ancient Exercises for Contemporary Citizens ", Educational Research and Reviews, n 11 (20), 2016, p. 1925-1930 ; J. Dainville \& B. Sans, «L'éloge paradoxal. Regards croisés sur deux expériences bruxelloises», dans P. Chiron \& B. Sans (dir.), Les progymnasmata en pratique de l'Antiquité à nos jours, Paris, Éditions Rue de l'Ulm, 2020, p. 356-372 (voir également, dans ce même volume, d'autres comptes rendus d'expériences en cours, en Europe et dans le monde, autour des exercices anciens).

B. Sans, «Exercer l'invention ou (ré)inventer la controverse", Exercices de rhétorique, $\mathrm{n}^{\circ} 5$ («Rhétorique et citoyenneté », sous la dir. de V. Ferry \& B. Sans), 2015. Disponible en ligne sur <https://journals.openedition.org/rhetorique/404> [consulté le 21 mars 2021]; B. Sans, «Des exercices anciens pour les citoyens de demain. Bilan d'un an d'enseignement de la rhétorique ", Enjeux, n 91, 2017, p. 113-135 ; B. Sans, « From Technique to Tactics: How to Teach Dispositio? ", dans ISSA 2018 Proceedings (actes du colloque d'Amsterdam, juillet 2018), Amsterdam, Sciental International Centre for Scholarship in Argumentation Theory, 2019, p. 1034-1041.

11. V. Ferry \& B. Sans, «L'intelligence émotionnelle : un art rhétorique », Le Langage et l'Homme, $\mathrm{n}^{\circ} 50,2,2015$, p. $147-161$. 
12. L'exercice dans son intégralité, tel qu'il a été présenté pour la première fois en septembre 2018 et ensuite repris dans le cadre de nos différentes formations (site du GRAL <http://gral.ulb.ac.be/>: catégories «Laboratoire de la démocratie » et «Formation polarisation »), est disponible en annexe de ce présent article.

13. Nous faisons ici référence, notamment aux travaux du prof. Vincent Donckier de Donceel sur la greffe de foie et les questions d'éthique (V. Donckier, et al., « Ethical Considerations Regarding Early Liver Transplantation in Patients With Severe Alcoholic Hepatitis not Responding to Medical Therapy », Journal of Hepatology, v.6 0, 2014, p. 866-871) mais également, à des questions qui se posent en des termes similaires dans le contexte de la pandémie de Covid-19. Voir à ce propos le regard que Michel Dupuis, professeur émérite à l'UC Louvain en philosophie et biomédicale, pose sur la crise sanitaire actuelle et les droits du patient (M. Dupuis, « Le droit du patient n'est possible que si les soignants sont dans des conditions acceptables » entretien par E. Burgraff, Le Soir, rubrique «Société ». Disponible en ligne sur <https://plus.lesoir.be/347249/ article/2021-01-05/michel-dupuis-le-droit-du-patient-nest-possible-que-si-les-soignants-sontdans> [consulté le 5 janvier 2021]).

14. Voir notamment : E. Danblon, « Régimes de rationalité, post-vérité et conspirationnisme : a-ton perdu le goût du vrai ? ", Argumentation et Analyse du Discours, n 25, 2020. Disponible en ligne sur: <https://doi.org/10.4000/aad.4528> [consulté le 24 mars 2021] ; C. Pieters, E. Danblon \& J.P. Laumond, «Roboticists and Reporters: A Rhetorical Experiment at the Cité des Sciences et de l'Industrie of Paris ", IEEE Robotics \& Automation Magazine, 2020. Disponible sur <10.1109/MRA. 2020.2994825> [consulté le 25 mars 2021].

15. B. Sans, "How to Create Rhetorical Exercises?", dans S. Oswald \& D. Maillat (dir.), Argumentation and Inference: Proceedings of the $2^{\text {nd }}$ European Conference on Argumentation (actes du colloque de Fribourg, 2017), Londres, College Publications, 2018, p. 717-730.

16. Il est important de souligner, à ce stade, que nous avons expressément présenté le foie comme une ressource limitée (un seul foie disponible) et une ressource compatible avec chacun des patients (ce qui est rarement le cas dans un contexte médical, mais qui n'est évidemment pas exclu).

17. Voir B. Sans, « How to Create Rhetorical Exercises? », art. cité.

18. Pour une présentation historique des lieux, voir L. Pernot, "Lieu et lieu commun dans la rhétorique antique ", Bulletin de l'Association Guillaume Budé, n 3, 1986, p. 253-284.

19. Nous empruntons les catégories suivantes à la théorie de Quintilien (III, 8) pour la construction d'un discours délibératif : les lieux ayant trait au registre pragmatique (l'utile, le faisable, le sûr, l'opportun, le nécessaire, le possible et la règle) et les lieux ayant trait au registre éthique et moral (justice, mérite, équité, clémence, gloire, etc.).

20. Voir B. Sans, «Exercer l'invention ou (ré)inventer la controverse », art. cité.

21. Voir B. Sans, « Exercer l'invention ou (ré)inventer la controverse », art. cité.

22. S. Di Piazza, F. Piazza \& M. Serra, "The Need for More Rhetoric in the Public Sphere. A Challenging Thesis About Post-Truth », Versus, $n^{\circ}$ 127/2, 2018, p. 225-242.

23. E. Danblon, «The Reason of Rhetoric ", Philosophy and Rhetoric, $n^{\circ} 46 / 4,2013$, p. 493-507.

24. E. Danblon, "Il y a critique et critique : épistémologie des modèles d'argumentation ", Argumentation et Analyse du Discours, n 9, 2012. Disponible sur <http://aad.revues.org/1395> [consulté le 21 mars 2021].

25. Ch. Perelman, L'Empire rhétorique, Paris, Vrin, 1977, p. 31. 


\section{RÉSUMÉS}

Cet article expose la conception de l'enseignement de la rhétorique à l'École de Bruxelles. Il explique l'origine de la démarche dans la pensée de Perelman. Il montre ensuite comment l'équipe du GRAL a retrouvé l'enseignement des exercices de rhétorique par les sophistes. Il propose une réflexion épistémologique pour montrer la spécificité de cet enseignement. Il termine enfin par l'exposé d'un exercice de délibération conçu et animé par les membres du GRAL.

This paper outlines the conception of the teaching of rhetoric at the Brussels School. It explains the origin of the approach in the thought of Perelman. It then shows how the GRAL team recovered the teaching of rhetorical exercises by the sophists. It proposes an epistemological reflection to show the specificity of this teaching. Finally, it concludes with the presentation of a deliberative exercise designed by the members of GRAL.

INDEX

Keywords : teaching of rhetoric, Perelman, exercises, sophists, deliberation

Mots-clés : enseignement de la rhétorique, Perelman, exercices, sophistes, délibération

\section{AUTEURS}

\section{EMMANUELLE DANBLON}

Université libre de Bruxelles, GRAL

Emmanuelle Danblon est professeure ordinaire à l'Université libre de Bruxelles, membre de l'Académie royale de Belgique (classe des lettres). Elle enseigne la rhétorique et l'argumentation. Ses domaines de recherche se centrent sur les liens entre les discours, les institutions et la rationalité. Elle dirige le GRAL (Groupe de recherche en rhétorique et argumentation linguistique) dans une perspective interdisciplinaire (<http://gral.ulb.ac.be>). Elle est secrétaire de la fondation Perelman de Bruxelles.

\section{LUCIE DONCKIER DE DONCEEL}

Université libre de Bruxelles, GRAL

Lucie Donckier est étudiante en deuxième année de doctorat, en cotutelle avec l'Università Degli Studi di Palermo (Eikos) et l'Université libre de Bruxelles (GRAL). Son travail de recherche porte sur les discours conspirationnistes et la notion de rationalité qu'elle étudie à travers le prisme de la rhétorique. Elle est également assistante chargée d'exercices à l'Université libre de Bruxelles.

\section{BENOÎT SANS}

Université libre de Bruxelles, GRAL

Benoît Sans est maître de conférences en latin à l'Université libre de Bruxelles (GRAL) et chercheur contractuel à l'université de Lille (laboratoire « STL », UMR 8163). Ses recherches portent sur les liens entre rhétorique et historiographie ancienne ( $\mathrm{I}^{\mathrm{r}}-\mathrm{III}{ }^{\mathrm{e}}$ siècles après $\mathrm{J} . \mathrm{-C}$.), ainsi que sur la réadaptation des exercices de rhétorique hérités de l'Antiquité à des publics et besoins contemporains. 


\section{BENJAMIN SEVESTRE-GIRAUD}

Aix-Marseille Université (CPAF) et Université libre de Bruxelles, GRAL

Benjamin Sevestre-Giraud est agrégé de lettres classiques et doctorant contractuel en rhétorique et sciences de l'Antiquité à Aix-Marseille Université (CPAF-TDMAM) et à l'Université libre de Bruxelles (GRAL). Ses recherches portent sur l'histoire de l'enseignement de la rhétorique et, plus spécifiquement, sur l'Institution oratoire de Quintilien et l'actualisation de la tradition et des pratiques rhétoriques dans l'enseignement secondaire et supérieur contemporain. 